\title{
Very Low Mass Stars and Brown Dwarf Candidates in Orion OB1a and OB1b
}

\author{
Frederick M. Walter \& William H. Sherry \\ Stony Brook University, Stony Brook NY 11794 \\ Scott J. Wolk \\ Center for Astrophysics, 60 Garden St, Cambridge MA 02138
}

\begin{abstract}
V R I$ images within the belt of Orion and the Ori OB1a association reveal a pre-main sequence locus extending to below our completeness limit of about $V=21$. We report here on followup JHK imaging and optical and near-IR spectroscopy of the faintest and reddest of the PMS candidates. We find that they are unreddened mid-to-late M "stars" which fall on a few million year isochrone. Masses are largely substellar, reaching as low as about $0.02 \mathrm{M}_{\odot}$ (20 Jovian masses). The space density of the substellar objects is high.
\end{abstract}

OB associations are fossil star forming regions (FSFRs; Blaauw 1991). They have ages of no more than a few tens of millions of years, because the $\mathrm{O}$ and $\mathrm{B}$ stars burn out quickly, and the associations are gravitationally unbound. Star formation is over, but all the products of the star formation process are still there. The dust and gas have largely dispersed, permitting an unobscured view. Accretion is over: all stars have attained their final masses. Few stars retain disks, simplifying interpretation of the photometric colors. Since most low mass stars form in OB associations, these FSFRs provide a more typical view of low mass star formation in our Galaxy than do the $\mathrm{T}$ associations.

Orion OB1, the largest nearby OB association, consists of four distinct subgroups (Blaauw 1964). Ori OB1a, the oldest and closest (Brown, de Geus, $\&$ de Zeeuw 1994) of the subassociations, has an age of about 10 million years (Myr). It is located north of Ori OB1b, the belt, with an age of 1-2 Myr. To the east of the belt is the imbedded star forming region NGC 2024. To the south, but considered part of OB1b, lies $\sigma$ Orionis. Ori OB1c surrounds, and is older than, Ori OB1d, the Orion Nebula Cluster (ONC), with an age of $<1 \mathrm{Myr}$ and active ongoing star formation ${ }^{1}$. Hillenbrand (1997) reported on its low mass population. Star formation in the Orion OB1 association has recently been reviewed by Brown, Walter, \& Blaauw (1998) and Walter et al. (2000).

The richest non-obscured FSFR in Orion studied to date is the $\sigma$ Ori cluster (Walter, Wolk, \& Sherry 1998). The X-ray images and an unbiased spectroscopic followup led to the identification of 104 likely pre-main sequence (PMS) stars within $30^{\prime}$ of $\sigma$ Ori. Walter et al. (1998) estimated a space density of 120

${ }^{1}$ OB1-Kenobi is in a galaxy far, far away. 
PMS stars per $\operatorname{deg}^{2}(10<\mathrm{V}<15)$, and about 700 PMS stars $/ \mathrm{deg}^{2} \quad(12<\mathrm{V}<19)$ from the photometric color-magnitude diagram (CMD). Wolk \& Walter (2000) showed that the pre-main sequence locus extended below the hydrogen burning limit. Béjar, Zapatero Osorio, \& Rebolo (1999) undertook a deeper photometric followup of this region, and confirmed the high space density of low mass PMS stars.

This is an update on a study of low mass stars in the Orion OB1 association, with emphasis on the faintest and lowest mass objects. Further details are reported elsewhere in this volume by Sherry et al.

\section{Substellar Mass Objects in Orion OB1}

The $\mathrm{OB}$ associations are attractive regions wherein to search for substellar mass objects. At young ages, these objects are relatively bright and hot, and therefore detectable with fairly modest instruments. Table 1 shows the predicted magnitudes, from the BCAH98 models (Baraffe et al 1998) of objects of 0.075 and 0.025 solar masses at the $440 \mathrm{pc}$ distance of Orion OB1b, for ages of 2,10 , and $100 \mathrm{Myr}$. Note that, although $10 \mathrm{Myr}$ corresponds to the age of the OB1a association, OB1a is closer to us than OB1b, and appears 0.6 mag brighter.

\begin{tabular}{|c|c|c|c|c|c|c|c|c|}
\hline \multicolumn{9}{|c|}{ Substellar Magnitudes in Orion (BCAH98) } \\
\hline & & 0.0 & $5 \mathbf{M}_{\odot}$ & & & 0.07 & $\mathbf{M}_{\odot}$ & \\
\hline age (Myr) & V & $\mathrm{K}$ & $\mathrm{V}-\mathrm{K}$ & $\mathrm{J}-\mathrm{K}$ & V & $\mathrm{K}$ & $\mathrm{V}-\mathrm{K}$ & $\mathrm{J}-\mathrm{K}$ \\
\hline 2 & 23.0 & 15.8 & 7.27 & 1.00 & 19.7 & 13.5 & 6.15 & 0.96 \\
\hline 10 & 23.7 & 16.3 & 7.50 & 1.01 & 20.8 & 15.1 & 5.70 & 0.93 \\
\hline 100 & 31.7 & 19.4 & 12.22 & 1.12 & 23.3 & 17.0 & 6.32 & 0.93 \\
\hline
\end{tabular}

Our data include:

- CTIO 0.9m CFIM BVRI images of about $4 \mathrm{deg}^{2}$ of Ori OB1, with completeness limits reaching $V=21$ in many areas, and $V=24$ in selected regions,

- IRTF/NSFCAM photometry of some of the reddest objects,

- CTIO/HYDRA multi-object spectra of selected fields, and

- IRTF/SPEX spectroscopy of some of the substellar mass candidates.

The optical CMD yields a mass function (MF) along the PMS locus that is indistinguishable from the field star MF (Kroupa 2002). Where we are complete, we find 150 stars $/ \mathrm{deg}^{2}\left(44 \mathrm{M}_{\odot} / \mathrm{deg}^{2}\right)$ between 0.21 and $0.65 \mathrm{M}_{\odot}$. Our deep fields show that the PMS locus does not terminate at the substellar mass limit, as has been noted by Wolk \& Walter (2000), Béjar et al. (1999), and Zapatero Osorio et al. (1999) in the $\sigma$ Ori region. Throughout the belt, we find a multitude of objects with $V>19$ and $V-I>3$. The near-IR photometry confirms that most of these objects have $V-K$ colors in the range 6-8 mag (see Fig. 1).

Comparison of the BVRIJHK spectral energy distributions (SEDs) with a grid of PMS isochrones from Baraffe et al (1998) yields the masses, ages, extinctions, and distance moduli. There is an ambiguity in using the optical photometry alone, as the reddening vector closely parallels the PMS locus. At this age, the more massive substellar mass objects have mid-M spectral types. We set out to confirm that the objects were indeed very low mass by obtaining spectra. First we obtained low dispersion spectra in the red of objects with 


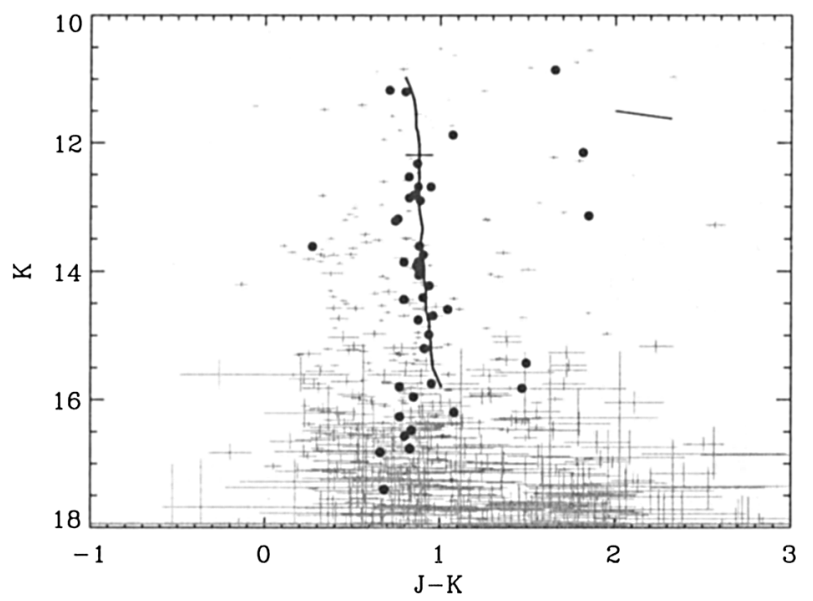

Figure 1. Near-IR CMD for very red objects in the belt of Orion. The approximately vertical track is Baraffe's (1998) 2 Myr isochrone. The horizontal line marks the substellar limit; the bottom of the isochrone is at 25 Jovian masses. The targets (solid circles), selected by $V-I$ colors, tend to lie near the isochrone, and are mostly substellar. The targets far to the right of the isochrone are reddened; the $A_{V}=1$ reddening vector is indicated. The grey bars are untargeted sources in the field. Galactic stars have $J-K \sim 0.5$.

$16<V<19$ using CTIO/HYDRA. These should all be bona fide stars. For the purposes of this report on substellar mass objects, the main result is a confirmation that the extinction is generally small: optical colors closely correspond to the spectral types. We also obtained low dispersion near-IR spectra of some of the fainter substellar mass objects. These indeed have spectral types in the M5-M7 range (Fig 2), consistent with their colors and expectations.

Fig. 1 shows that the data are consistent with a large substellar mass population. Since the candidates are selected on the basis of optical colors, they do not form an unbiased sample and cannot be used to estimate the space density of substellar mass objects. However, from the 8 substellar mass objects confirmed in $0.15 \mathrm{deg}^{2}$ of Orion OB1b, we can place a lower limit on the space density of substellar mass objects $\left(0.075>\frac{\mathrm{M}}{\mathrm{M}_{\odot}}>0.06\right)$ of about 50 per $\operatorname{deg}^{2}$.

\section{Conclusions}

This investigation verifies that there is indeed a high density of substellar mass objects in Orion OB1a and OB1b. We are proceeding to quantify the completeness limits and hence the true density of substellar mass objects. A major effort is underway now to separate the populations of the OB1b and the older but nearer OB1a associations. We are also studying the spatial variations of the $\mathrm{MF}$, with an aim to studying the mass segregation in this association, and perhaps to determine whether the substellar mass objects are ejected from compact cores before they have a chance to accrete up to stellar masses. 


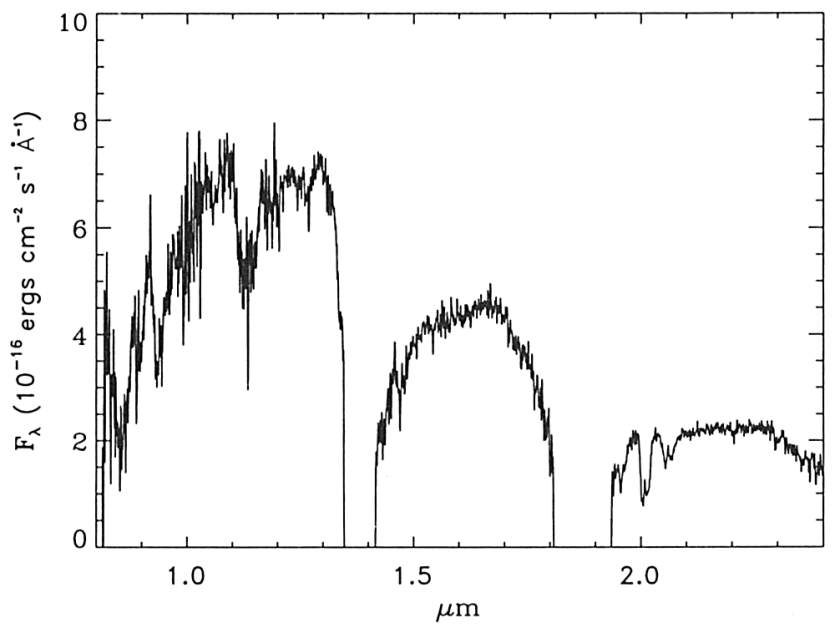

Figure 2. The near-IR spectrum from the IRTF/SPEX of one of the stars in our sample. This star, near $\epsilon$ Orionis, has $K=13.5$ and $V-K=7.6$. The best fit mass and age are $0.06 \pm 0.02 \mathrm{M}$, and $1 \mathrm{Myr}$, based on the VRIJHK SED. The spectral type is M7.

\section{References}

Baraffe, I., Chabrier, G., Allard, F., \& Hauschildt, P.H. 1998, A\&A, 337, 403

Béjar,V.J.S., Zapatero Osorio, M.R., \& Rebolo, R., 1999, ApJ, 521, 671

Blaauw, A. 1964, ARA\&A, 2, 213

Blaauw, A. 1991, in NATO ASI Vol. 345, The Physics of Star Formation and Early Stellar Evolution, ed. C.J. Lada \& N.D. Kyfalis, 125

Brown, A.G.A., de Geus, E.J., \& de Zeeuw, P.T., 1994, A\&A, 289, 101

Brown, A.G.A., Walter, F.M., \& Blaauw, A. 1998, in The Orion Complex Revisited, ed. A. Burkert \& M. McCaughrean, in press

Hillenbrand, L., 1997, AJ, 113, 1733

Kroupa, P. 2002, Science, 295, 82

Walter, F.M., et al., 2000, in Protostars and Planets IV, ed. V. Mannings, A.P. Boss, \& S.S. Russell (Tucson: University of Arizona), 273

Walter, F.M., Wolk, S.J., \& Sherry, W.H. 1998, in ASP Conf. Ser. Vol. 154, Cool Stars, Stellar Systems, and the Sun, ed. R.A. Donahue \& J.A. Bookbinder (San Francisco: ASP), CD-1793

Wolk, S.J. \& Walter, F.M. 2000, in Proceedings of the Euroconference on Very Low Mass Stars and Brown Dwarfs in Stellar Clusters and Associations, ed. R. Rebolo \& M.R. Zapatero Osorio, 38

Zapatero Osorio, M.R., Béjar, V.J.S., Rebolo, R., Martín, E.L., \& Basri, G., 1999, ApJ, 524, L115 